\title{
AVALIAÇÃO DA ATIVIDADE ANTIMICROBIANA DOS ÓLEOS ESSENCIAIS DE THYMUS VULGARIS (TOMILHO), SYZYGIUM AROMATICUM (CRAVO-DA-ÍNDIA) E ROSMARINUS OFFICINALIS (ALECRIM) E DOS CONSERVANTES BENZOATO DE SÓDIO E SORBATO DE POTÁSSIO EM ESCHERICHIA COLI E STAPHYLOCOCCUS AUREUS
}

\author{
Angela Aparecida da Silva ${ }^{1 *}$ \\ Márcia Maria dos Anjos ${ }^{2 * *}$ \\ Suelen Pereira Ruiz ${ }^{2 *}$ \\ Lucimara Bergamo Panice ${ }^{3 * * *}$ \\ Jane Martha Graton Mikcha $a^{4 * * * *}$ \\ Miguel Machinski Junior ${ }^{5 * * * * *}$ \\ Benício Alves de Abreu Filho $5^{\text {tr+m+n}}$
}

\begin{abstract}
Neste trabalho foi avaliada a ação dos óleos essenciais de Thymus vulgaris (tomilho), Syzygium aromaticum (cravo-da-índia) e Rosmarinus officinalis (alecrim) e dos conservantes benzoato de sódio e sorbato de potássio como agentes antimicrobianos. As cepas de Staphylococcus aureus (ATCC 25923) e Escherichia coli (ATCC 25922) foram utilizadas no teste de susceptibilidade antimicrobiana usando-se a técnica de microdiluição em microplaca de 96 poços para avaliação da Concentração Inibitória Mínima (CIM) e, posteriormente, subcultivo em Mueller Hinton Agar para avaliação da Concentração Bactericida Mínima (CBM). As concentrações dos óleos e conservantes sintéticos testados variaram de 15,6 a $1000 \mu \mathrm{g} / \mathrm{mL}$. As microdiluições utilizando inóculos bacterianos nas concentrações de $10^{4} \mathrm{UFC} / \mathrm{mL}$ foram incubadas a $37^{\circ} \mathrm{C} / 24 \mathrm{~h}$. As CIM para os óleos essenciais de cravo, tomilho e alecrim foram de 550, 650 e $>1000 \mu \mathrm{g} / \mathrm{mL}$ para E. coli e 550, 800 e $1000 \mu \mathrm{g} / \mathrm{mL}$ para $S$. aureus, respectivamente. No entanto, a ação bactericida dos óleos essenciais do cravo-da-índia e do tomilho foi encontrada apenas em relação a $E$. coli, na concentração de 550 e $850 \mu \mathrm{g} / \mathrm{mL}$, respectivamente. Para os dois conservantes sintéticos testados, a CIM foi $>1000 \mu \mathrm{g} / \mathrm{mL}$, portanto não apresentaram atividade antibacteriana contra os microrganismos testados. Trabalhos futuros deverão ser realizados para verificar a eficiência dos antimicrobianos naturais anteriormente citados e para avaliar a possibilidade de serem utilizados na indústria de alimentos.
\end{abstract}

PALAVRAS-CHAVE: ÓLEOS ESSENCIAIS, ATIVIDADE ANTIBACTERIANA, CONSERVANTES.

*1 Mestranda em Ciência de Alimentos, Universidade Estadual de Maringá (UEM), Maringá, PR (e-mail: angela-bio1@hotmail.com).

**2 Doutorandas em Ciência de Alimentos, Universidade Estadual de Maringá (UEM), Maringá, PR (e-mail: marciamanjos@hotmail.com; suelen_ruiz@hotmail.com).

***3 Professora Adjunta, Departamento de Ciências Biológicas, [Extenso] (FAFIMAN), [Cidade], [Estado] (e-mail: lucimarabergpan@hotmail.com).

****4 Professora Associada, Departamento de Análises Clínicas e Biomedicina, Universidade Estadual de Maringá (UEM), Maringá, PR (e-mail: jmgmikcha@uem.br).

*****5 Professores Associados, Departamento de Ciências Básicas da Saúde, Universidade Estadual de Maringá (UEM), Maringá, PR (e-mail: machinski.jr@gmail.com; baafilho@gmail.com). 


\section{INTRODUÇÃO}

O uso de produtos naturais e seus derivados nas indústrias de alimentos, farmacêutica, cosmética e química tem aumentado devido a fatores como a proliferação microbiana e sua multirresistência aos antibióticos e aos agentes químicos sintéticos. Há vários mecanismos de tratamento e controle microbiológico que podem inibir ou matar microrganismos, que abrangem agentes físicos, químicos ou uma combinação de ambos. Em certas situações, o tratamento tornase ineficaz devido à presença de microrganismos resistentes, fato que leva à necessidade de desenvolvimento de novas formas de controle microbiano (STANOJEVIC et al., 2009). O National Committee for Clinical Laboratory Standards (NCCLS) (2003) relaciona a resistência microbiana a fatores como a produção de enzimas que inativam os antimicrobianos, alterações do sítio-alvo desses fármacos e alteração da absorção ou do efluxo desses agentes.

$\mathrm{Na}$ indústria alimentícia, a deterioração dos alimentos por microrganismos - como a oxidação de lipídios, que provoca a rancificação, deixando o alimento com odores e sabores desagradáveis, além de causar a formação de produtos secundários tóxicos (PROESTOS; ZOUMPOULAKIS; SINANOGLOU, 2013) - altera a qualidade dos produtos. O uso de agentes antimicrobianos sintéticos como o benzoato de sódio e o sorbato de potássio retarda o processo de deterioração dos alimentos causado por microrganismos, aumentando seu tempo de prateleira (STANOJEVIC et al., 2009). No entanto, a pesquisa com produtos naturais tem buscado substituir esses agentes sintéticos, uma vez que óleos essenciais extraídos de plantas fenólicas apresentam grande potencial bacteriostático ou bactericida devido à presença de compostos bioativos, como os polifenóis (PROESTOS; ZOUMPOULAKIS; SINANOGLOU, 2013).

Segundo a Agência Nacional de Vigilância Sanitária (ANVISA) (2013), no Brasil, o S. aureus é o segundo microrganismo mais relacionado a casos de surtos registrados entre os anos de 2000 e 2013. São 759 casos registrados, estando atrás somente da Salmonella spp., com 1522 casos registrados. A E. coli ocupa o quarto lugar, com 475 casos.

Em nível mundial, no ano de 2011 ocorreram aproximadamente 9,4 milhões de casos de doenças transmitidas por alimentos, causadas por 31 agentes patogênicos conhecidos. Entre eles, oito foram responsáveis pela maioria dos casos que levaram a hospitalizações e mortes. Dentre esses agentes estão o Staphylococcus aureus e a Escherichia coli (STEC) 0157, que, portanto, são os microrganismos mais utilizados como referência em testes antimicrobianos.

Este trabalho determinou a Concentração Inibitória Mínima (CIM) e a Concentração Bactericida Mínima (CBM) dos óleos essenciais de Thymus vulgaris (tomilho), Syzygium aromaticum (cravo-da-índia) e Rosmarinus officinalis (alecrim), bem como dos conservantes benzoato de sódio e sorbato de potássio em Staphylococcus aureus (ATCC 25923) e Escherichia coli (ATCC 25922).

\section{MATERIAL E MÉTODOS}

\subsection{MEIOS DE CULTURA}

Os meios de cultura utilizados foram adquiridos comercialmente e preparados conforme recomendado pelo fabricante, sendo em seguida autoclavados a $121^{\circ} \mathrm{C} / 15 \mathrm{~min}$. Utilizou-se o meio de Mueller Hinton Broth (Difco) nas microdiluições em microplaca para avaliação da CIM e Mueller Hinton Agar (Difco) nos microcultivos para avaliação da CBM.

O Mueller Hinton Agar é recomendado pelo NCCLS (2003) para testes de sensibilidade contra bactérias não fastidiosas, facultativas ou aeróbias, e quando suplementados podem ser utilizados em bactérias fastidiosas. Isto se deve à sua reprodutividade entre os lotes nos testes, aos teores baixos de inibidores de sulfonamida, trimetoprim e tetraciclina, ao crescimento satisfatório da maioria dos patógenos, e ao grande acervo de sua utilização para esse procedimento. 


\section{2 ÓLEOS ESSENCIAIS}

Os óleos essenciais foram obtidos mediante extração de folhas secas de tomilho, alecrim e cravo (Thymus vulgaris L., Rosmarinus officinalis e Syzygium aromaticum - fornecidos pela Nutricrok Alimentos Ltda, Maringá, PR, Brasil) pelo processo de hidrodestilação, utilizando-se o aparelho Clevenger, segundo o método descrito pela Farmacopeia Europeia (COUNCIL OF EUROPE, 1997).

Os óleos essenciais foram mantidos refrigerados à temperatura de $3^{\circ} \mathrm{C}$ e protegidos da luz até o momento de uso (BURT, 2004). Realizou-se a caracterização dos óleos essenciais de T. vulgaris e R. officinalis por Ressonância Magnética Nuclear (RMN) e por Cromatografia em fase gasosa acoplado a espectrômetro de massas (CG-MS) no Laboratório de Toxicologia da Universidade Estadual de Maringá (UEM). Os dois componentes majoritários encontrados no óleo essencial de T. vulgaris foram o borneol, com 40,6\%, e o a-repineol, com 19,9\%. Para o óleo essencial do R. officinalis, os dois componentes majoritários foram 1,8-cineol, com 52,2\%, e o camphor, com $15,2 \%$. Não foi possível caracterizar o óleo essencial do $S$. aromaticum.

Antes de serem utilizados nas microdiluições em placa, os óleos essenciais foram diluídos com dimetilsulfóxido (DMSO), permanecendo em concentração final de $5 \%$ do volume total da solução (meio+solubilizante), e em seguida foram adicionados ao meio de cultura para realização do teste de susceptibilidade antimicrobiana (AL-BAYATI, 2008).

\subsection{CONSERVANTES}

O sorbato de potássio e o benzoato de sódio foram adquiridos da Vetec® (Rio de Janeiro, Brasil), sendo preparadas soluções aquosas de $2000 \mu \mathrm{g} / \mathrm{mL}$ para ambos os conservantes sintéticos.

\subsection{PREPARAÇÃO DO INÓCULO E DA SUSPENSÃO BACTERIANA}

As bactérias Staphylococcus aureus (ATCC 25923) e Escherichia coli (ATCC 25922) foram obtidas no banco de microrganismos do laboratório de Microbiologia da UEM para utilização no teste de susceptibilidade antimicrobiana, sendo ativadas em caldo Mueller Hinton e incubadas a $37^{\circ} \mathrm{C} / 24 \mathrm{~h}$.

Preparou-se a suspensão bacteriana 15 minutos antes de ser inoculada nos poços, cujo inóculo foi padronizado conforme recomenda o NCCLS (2003). Primeiramente realizou-se a suspensão bacteriana em solução salina a $0,85 \%$ para obter turbidez equivalente à solução

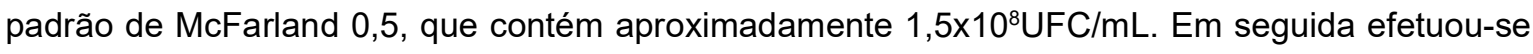
diluição 1:10 em caldo Mueller Hinton, deixando a suspensão bacteriana a uma concentração de $10^{7} \mathrm{UFC} / \mathrm{mL}$ para que ao término de todas as microdiluições, ao acrescentar $5 \mu \mathrm{L}$ do inóculo em cada poço (contendo o meio de cultura e o agente antimicrobiano), a concentração final estivesse em aproximadamente $5 \times 10^{4} \mathrm{UFC} / \mathrm{mL}$.

\subsection{AVALIAÇÃO DA CONCENTRAÇÃO INIBITÓRIA MÍNIMA (CIM) E DA CONCENTRAÇÃO BACTERICIDA MÍNIMA (CBM)}

A Concentração Inibitória Mínima (CIM) foi determinada através da microdiluição seriada em microplacas de 96 poços (TPP ${ }^{\circledR}$ ), observando-se diretamente a olho nu o crescimento ou não do inóculo mediante turvação do meio de cultura presente no poço. A análise de CIM e CBM foi realizada conforme estabelece o NCCLS (2003).

O poço límpido foi avaliado como sendo a menor concentração do agente testado capaz de inibir o crescimento e o desenvolvimento do microrganismo. A partir dele foram realizadas microculturas em placas de Petri com Mueller Hinton Ágar pelo plaqueamento de $10 \mu \mathrm{L}$ dos poços negativos e positivos para avaliação da CBM (OKOH; SADIMENKO; AFOLAYAN, 2010). A placa de Petri foi incubada a $37^{\circ} \mathrm{C} / 24 \mathrm{~h}$ para observação do crescimento de colônias, avaliando-se assim 
a eficiência do agente teste: a ausência da proliferação de colônias da bactéria indica que a droga apresentou ação bactericida, enquanto o crescimento de colônias indica que o agente antimicrobiano mostrou apenas ação bacteriostática. Todos os experimentos foram realizados em triplicata.

\section{RESULTADOS E DISCUSSÃO}

De acordo com os resultados obtidos nos ensaios de susceptibilidade antimicrobiana, os óleos essenciais apresentaram melhores resultados frente às bactérias $E$. coli e $S$. aureus quando comparados aos tratamentos com os conservantes benzoato de sódio e sorbato de potássio, como demonstra a Tabela 1:
TABELA 1 - CONCENTRAÇÃO INIBITÓRIA MÍNIMA (CIM) E CONCENTRAÇÃO BACTERICIDA MÍNIMA (CBM) DAS SUBSTÂNCIAS AVALIADAS NO TESTE DE SUSCEPTIBILIDADE ANTIMICROBIANA PARA E. COLI E S. AUREUS

\begin{tabular}{|l|c|c|c|}
\hline \multirow{3}{*}{ CEPA BACTERIANA } & TRATAMENTO & CIM $(\boldsymbol{\mu g} / \mathbf{m L})$ & CBM $(\boldsymbol{\mu g} / \mathbf{m L})$ \\
\hline \multirow{3}{*}{ Escherichia coli } & Sorbato de potássio & $>1000$ & $>1000$ \\
& Benzoato de sódio & $>1000$ & $>1000$ \\
& OE Thymus vulgaris & 650 & 850 \\
& OE Syzygium aromaticum & 550 & 550 \\
& OE Rosmarinus officinalis L. & $>1000$ & $>1000$ \\
\hline \multirow{5}{*}{ Staphylococcus aureus } & Sorbato de potássio & $>1000$ & $>1000$ \\
& Benzoato de sódio & $>1000$ & $>1000$ \\
& OE Thymus vulgaris & 800 & $>1000$ \\
& OE Syzygium aromaticum & 550 & $>1000$ \\
& OE Rosmarinus officinalis L. & $>1000$ & $>1000$ \\
\hline
\end{tabular}

OE: Óleo Essencial; (>): maior que.

Holetz et al. (2002) classificaram os agentes antimicrobianos conforme a concentração apresentada, desempenhando: boa atividade antimicrobiana quando suas concentrações estão menores que $500 \mu \mathrm{g} / \mathrm{mL}$; baixa atividade antimicrobiana quando as concentrações encontram-se entre $500-1000 \mu \mathrm{g} / \mathrm{mL}$; sem atividade antimicrobiana quando apresenta concentrações acima de $1000 \mu \mathrm{g} / \mathrm{mL}$.

Nos ensaios com o óleo essencial de $S$. aromaticum, os resultados encontrados para a CIM em relação a $E$. coli e $S$. aureus foi de $550 \mu \mathrm{g} / \mathrm{mL}$ para ambas, sendo este o melhor resultado entre todas as avaliações realizadas. No entanto, conforme a classificação previamente indicada, o óleo essencial de $S$. aromaticum apresentou baixa ação antimicrobiana. No trabalho apresentado por Burt (2004), o cravo-da-índia também apresentou melhor resultado como agente antimicrobiano quando comparado aos tratamentos com óleo essencial do tomilho e alecrim.

Para o tratamento com o óleo essencial de R. officinalis L. (alecrim), a CIM observada foi $>1000 \mu \mathrm{g} / \mathrm{mL}$ para as duas cepas bacterianas avaliadas. No trabalho realizado por Jordán et al. (2013) foram avaliados os principais constituintes encontrados nos óleos essenciais de diferentes quimiotipos de Rosmarinus officinalis L., sendo eles o eucaliptol, o cânfora e o a-pineno, que apresentaram diferenças percentuais entre seus constituintes dentro da mesma espécie. Esses 
dados diferem do encontrado em nossos ensaios, que teve como principais constituintes o 1,8-cineol, com $52,2 \%$, e a cânfora, com $15,2 \%$. Essas diferenças encontradas nos constituintes dos óleos essenciais podem estar relacionadas ao habitat ou ao clima, podendo existir correlação entre os diferentes métodos de extração. Jordán et al. (2013) obtiveram bons resultados com o óleo essencial de Rosmarinus officinalis L. como agente antimicrobiano frente a quatro agentes patogênicos de origem alimentar. No entanto, esses resultados apresentaram diferenças entre a ação dos variados quimiotipos, sugerindo que podem estar relacionados às variações encontradas nos constituintes dos óleos essenciais, assim como a favorável atividade antimicrobiana contra $E$. coli foi relacionada ao elevado teor de eucaliptol.

A CIM do óleo essencial de T. vulgaris foi $800 \mu \mathrm{g} / \mathrm{mL}$ para S. aureus e $650 \mu \mathrm{g} / \mathrm{mL}$ para E. coli. Diferentes resultados foram obtidos por Al-Bayati (2008), que constatou menor CIM para cepas de $S$. aureus em relação a $E$. coli. No entanto, deve-se levar em consideração a constituição química dos óleos essenciais, pois apresentam porcentagens diferentes de seus isolados, inclusive dentro da mesma espécie. O óleo essencial extraído do gênero Thymus apresenta ação antimicrobiana, podendo ser usado como conservante de alimentos, antisséptico, expectorante, vermífuga, entre outros. Seu princípio ativo está relacionado aos compostos fenólicos presentes em sua composição (BEHBAHANI et al., 2013). Rota et al. (2008) verificaram que o óleo essencial de T. vulgaris apresenta ação antimicrobiana

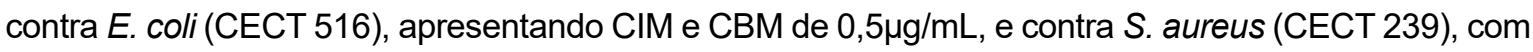
$\mathrm{CIM}$ e CBM de $<0,2 \mu \mathrm{g} / \mathrm{mL}$. No entanto, seu maior representante foi o timol com $57,7 \%$.

Os resultados para $E$. coli no ensaio da avaliação da CBM frente aos óleos essenciais de $S$. aromaticum e $T$. vulgaris foram de 550 e $850 \mu \mathrm{g} / \mathrm{mL}$, respectivamente, com diminuição de 7 log UFC/ $\mathrm{mL}$. Para $R$. officinalis, a CBM foi $>1000 \mu \mathrm{g} / \mathrm{mL}$, portanto não apresentou resultado antimicrobiano contra $E$. coli neste ensaio.

Para S. aureus, a CBM foi $>1000 \mu \mathrm{g} / \mathrm{mL}$ para todos os óleos essenciais avaliados, ainda que tenha havido redução da carga bacteriana com a utilização do tratamento. Para o tratamento com óleo essencial de $S$. aromaticum e $T$. vulgaris na concentração de $1000 \mu \mathrm{g} / \mathrm{mL}$, a redução foi de $1,12 \log U F C / m L$ e $1,13 \log U F C / m L$ respectivamente. Já o óleo essencial de $R$. officinalis não apresentou atividade antimicrobiana neste ensaio.

Devido à sua origem natural, os óleos essenciais contribuem para a qualidade e segurança dos alimentos, podendo ser utilizados como agentes antibacterianos (BURT, 2004) e como aromatizantes, além de serem melhor aceitos pelos consumidores (BEVILACQUA; SINIGAGLIA; CORBO, 2008). Da mesma maneira, o Código de Regulamentação Federal do FDA (21CFR182.20) (2013) estabelece que os óleos essenciais, as oleorresinas (sem solventes) e os extratos naturais (incluindo destilados) são Geralmente Reconhecidos como Seguros (GRAS - Generally Recognized as Safe) para o uso.

Em relação aos conservantes, o benzoato de sódio e o sorbato de potássio apresentaram $\mathrm{CIM} \mathrm{e} \mathrm{CBM}>1000 \mu \mathrm{g} / \mathrm{mL}$ tanto para $E$. coli quanto para $S$. aureus, não demonstrando bons resultados.

A ANVISA (2011) estabelece o limite máximo de $2000 \mu \mathrm{g} / \mathrm{mL}$ para o benzoato de sódio e sorbato de potássio em algumas categorias alimentares e, por serem conservantes, é necessário que as concentrações dessas substâncias estejam de acordo com a legislação, não podendo ser utilizadas acima da concentração permitida. Por isso, existe a necessidade de se buscar novos compostos com potencial antimicrobiano para auxiliar na ação dos conservantes.

\section{CONCLUSÃO}

Durante este trabalho, os óleos essenciais de tomilho e cravo-da-índia apresentaram atividade antimicrobiana para E. coli e $S$. aureus. Porém, existe a necessidade de se realizar novas pesquisas com essas substâncias em relação a outros microrganismos, assim como avaliar a ação antagônica ou sinérgica presente na combinação de diversos tratamentos com variados óleos essenciais. 


\section{EVALUATION OF ANTIMICROBIAL ACTIVITY OF THE ESSENTIAL OILS OF THYMUS VULGARIS (THYME), SYZYGIUM AROMATICUM (CLOVE) AND ROSMARINUS OFFICINALIS (ROSEMARY) AND THE PRESERVATIVES SODIUM BENZOATE AND POTASSIUM SORBATE IN ESCHERICHIA COLI AND STAPHYLOCOCCUS AUREUS}

This study evaluated the action of the essential oils of Thymus vulgaris (thyme), Syzygium aromaticum (clove) and Rosmarinus officinalis (rosemary) and the preservatives sodium benzoate and potassium sorbate as antimicrobial agents. Strains of Staphylococcus aureus (ATCC 25923) and Escherichia coli (ATCC 25922) were used in antimicrobial susceptibility testing via the microdilution technique performed in a 96-well microplate to evaluate the Minimum Inhibitory Concentration (MIC) and later in Mueller Hinton Agar subculture to review the Minimum Bactericidal Concentration (MBC). The concentrations of the oils and synthetic preservatives tested ranged from 15.6 to $1000 \mu \mathrm{g} / \mathrm{mL}$. The bacterial inocula microdilutions were used at concentrations of $10^{4} \mathrm{CFU} / \mathrm{mL}$ and incubated at $37^{\circ} \mathrm{C}$ for 24 hours. The MICs for the essential oils of clove, thyme and rosemary were 550, 650 and $>1000 \mu \mathrm{g} / \mathrm{mL}$ for $E$. coli and 550,800 and $1000 \mu \mathrm{g} / \mathrm{mL}$ for S. aureus, respectively. However, the bactericidal effect of the essential oils of clove and thyme was found only for $E$. coli in a concentration of 550 and $850 \mu \mathrm{g} / \mathrm{mL}$, respectively. For both synthetic preservatives tested, the MIC was $>1000 \mu \mathrm{g} /$ $\mathrm{mL}$; therefore, they showed no antibacterial activity against the microorganisms tested. Future work should be conducted to verify the efficiency of the above natural antimicrobials and to evaluate the possibility of their use in the food industry.

KEYWORDS: ESSENTIAL OILS, ANTIBACTERIAL ACTIVITY, PRESERVATIVES.

\section{REFERÊNCIAS}

1 AL-BAYATI, F. A. Synergistic antibacterial activity between Thymus vulgaris and Pimpinella anisum essential oils and methanol extracts. Journal of Ethnopharmacology, v. 116, p. 403-406, 2008.

2 ANVISA. Agência Nacional de Vigilância Sanitária. [título]. [ano]. Disponível em: <http://portal.anvisa.gov.br/wps/ wcm/connect/a6809d8047457a1c86c0d63fbc4c6735/Compendio marco 2011.pdf?MOD=AJPERES>. Acesso em: $02 / 02 / 2014$.

3 BEHBAHANI, M. H.; et al. Volatile oil composition and antimicrobial activity of two Thymus species. Pharmacognosy Journal, v.5, p. 77-79, 2013.

4 BEVILACQUA, A.; SINIGAGLIA, M.; CORBO, M. R. Alicyclobacilus acidoterrestris: New methods for inhibiting spore germination. International Journal of Food Microbiology, v. 125, p. 103-110, 2008.

5 BURT, S. Essential oils: their antibacterial properties and potential applications in foods - a review. International Journal of Food Microbiology, v. 94, p. 223-253, 2004.

6 COUNCIL OF EUROPE. Methods of Pharmacognosy. In European Pharmacopoeia, 1997. p.121-122.

7 EUROPEAN PARLIAMENTAND COUNCIL DIRECTIVE N 95/2/EC. On food additives other colours and sweeteners. 20 February 1995. Disponível em: <http://ec.europa.eu/food/fs/sfp/addit flavor/flav11 en.pdf>. Acesso em: 02/08/2013.

8 FDA. Food and Drug Administration. Code of Federal Regulations Title 21- Food and Drugs. Chapter I - Food and Drug Administration. Department of Health and Human Services - Subchapter B - Food for Human Consumption (continued). Part 182 - Substances Generally Recognized as Safe. Subpart A- General Provisions. Sec. 182.20. volume 3. Revised as of April 1, 2013. Disponível em: <http://www.accessdata.fda.gov/scripts/cdrh/cfdocs/cfcfr/CFRSearch.cfm?fr=182.20>. Acesso em: 02/08/2013.

9 HOLETZ, F. B.; et al. Screening of some plants used in the Brazilian folk medicine for the treatment of infectious diseases. Memórias do Instituto Oswaldo Cruz, v. 97, p.1027-1031, 2002.

10 JORDÁN, M. J.; et al. Effect of bioclimatic area on the essential oil composition and antibacterial activity of Rosmarinus officinalis L. Food Control, v. 30, p. 463-468, 2013. 
11 NATIONAL COMMITTEE FOR CLINICAL LABORATORY STANDARDS. Methods for Dilution Antimicrobial Susceptibility Tests for Bacteria That Grow Aerobically; Approved Standard - Sixth Edition. NCCLS document M7- A6 [ISBN 1-56238-486-4], 2003.

$12 \mathrm{OKOH}$, O. O.; SADIMENKO, A. P.; AFOLAYAN, A. J. Comparative evaluation of the antibacterial activities of the essential oils of Rosmarinus officinalis L. obtained by hydrodistillation and solvent free microwave extraction methods. Food Chemistry, v. 120, p. 308-312, 2010.

13 PROESTOS, C.; ZOUMPOULAKIS, P.; SINANOGLOU, V. J. Determination of plant bioactive compounds, antioxidant capacity and antimicrobial screening. Focusing on Modern Food Industry, v. 2, n.1, [página], 2013.

14 ROTA, M. C.; et al. Antimicrobial activity and chemical composition of Thymus vulgaris, Thymus zygis and Thymus hyemalis essential oils. Food Control, v. 19, p. 681-687, 2008.

15 STANOJEVIC, D.; et al. Antimicrobial effects of sodium benzoate, sodium nitrite and potassium sorbate and their synergistic action in vitro. Bulgarian Journal of Agricultural Science, v. 15, n. 4, p. 307-311, 2009.

\section{AGRADECIMENTOS}

Os autores agradecem a Pró-Reitoria de Pós-Graduação (PPG) da Universidade Estadual de Maringá e a Coordenação de Aperfeiçoamento de Pessoal de Nível Superior (CAPES). 